\title{
Diakoptic Solution of Induction Problems
}

\author{
C. R. BREWITt-TAYLOR and P. B. Johns \\ Department of Electrical Engineering, Nottingham University, Nottingham, U. K.
}

(Received November 3, 1977; Revised January 17, 1978)

\begin{abstract}
Two-dimensional induction problems are solved by means of an equivalent electrical network, which is then solved by the method of diakoptics. The network is divided into several subnetworks, which are partially solved independently of each other, and then are re-assembled into the full network. The advantages are that the size of arrays handled is reduced, and that it is possible to solve a problem which has most of the subnetworks the same as a previous problem more quickly than by solution without subdivision, since the common subnetworks need not be re-solved. A computer program has been written to use this method, and an example is given with storage and time used.
\end{abstract}

\section{Introduction}

The problem to be considered is the usual two-dimensional induction problem, in which fields and conductivities vary only in the $y$ and $z$ directions, and the fields vary with an assumed $\exp (i \omega t)$ time variation. In this case Maxwell's equations reduce to the wellknown diffusion type of equations for the two separate polarisation cases. In $E$-polarisation:

$$
\frac{\partial^{2} E_{x}}{\partial y^{2}}+\frac{\partial^{2} E_{x}}{\partial z^{2}}=i \omega \mu_{0} \sigma E_{x}
$$

and in $H$-polarisation:

$$
\frac{\partial}{\partial y}\left(\frac{1}{\sigma} \frac{\partial B_{x}}{\partial y}\right)+\frac{\partial}{\partial z}\left(\frac{1}{\sigma} \frac{\partial B_{x}}{\partial z}\right)=i \omega \mu_{0} B_{x} .
$$

Here all the symbols have their usual meanings. In these equations we are making the usual geophysical assumptions of negligible displacement currents and non-magnetic materials, though the method described in this paper can be simply extended to remove these assumptions.

It is well known that there is an analogy between Maxwell's equations in one or two dimensions and the equations relating voltage and current in a transmission line or surface; and similarly that a five-point difference approximation to the differential equations above has the same form as the equation describing an electrical mesh network of the kind shown in Fig. 1. This is discussed in a geophysical context by SwIFT (1971). In the present work the components of the network are considered to outline rectangles in each of which the conductivity is constant, and the difference equations represented are those of BrewitT-TAYLOR and WeAver (1976). In the E-polarisation case the voltages 


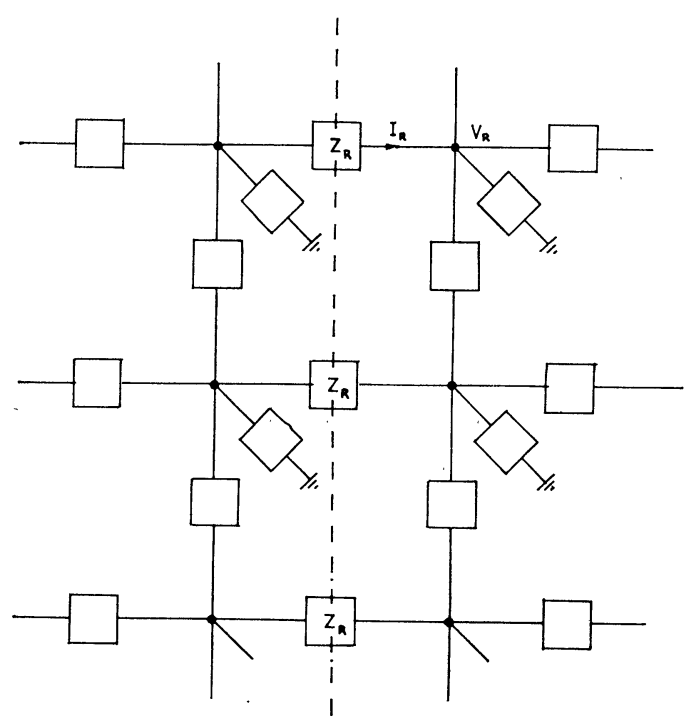

Fig 1. Part of the network representing the differential equation. The dashed line shows part of a diakoptic subnetwork boundary.

at the junctions of the network represent the electric field $E_{x}$; for a regular mesh of distance $h$ between junctions, the transverse components are inductors of value $\mu_{0}$ Henry, and the components to ground are resistors of value $1 / h^{2} \sigma$ Ohms. In the $H$-polarisation case the junction voltages represent the magnetic field $B_{x}$; the transverse components are resistors of value $\sigma \mathrm{Ohms}$, and the components to ground are capacitors of value $1 / h^{2} \mu_{0}$ Farads. In the case of uneven mesh spacings and conductivity boundaries, suitably averaged values of conductivity are used. However the diakoptic method described here does not depend on the values of the components, but only on the structure of the network, and so it may be used for either polarisation case. Both are special cases of the network in Fig. 1. In this network the voltages at adjacent junctions obey the following equation, which follows from Ohm's law and the conservation of current:

$$
\frac{\left(V_{1}-V_{0}\right)}{Z_{1}}+\frac{\left(V_{2}-V_{0}\right)}{Z_{2}}+\frac{\left(V_{3}-V_{0}\right)}{Z_{3}}+\frac{\left(V_{4}-V_{0}\right)}{Z_{4}}+\frac{V_{0}}{Z_{0}}=0
$$

where $V_{1}$ to $V_{4}$ are the voltages at the junctions surrounding a typical junction with voltage $V_{0}, Z_{1}$ to $Z_{4}$ are the corresponding transverse impedances and $Z_{0}$ is the impedance from the central point to ground.

Diakoptics is a general method for the solution of large electrical networks, originated by KRON (1963); a useful introduction is given by BRAMELLER et al. (1969). The method proceeds by dividing up the network into a number of subnetworks, which are analysed separately. For each subnetwork we analyse its internal properties, and then set up a matrix which describes it as it is seen from the outside along the branches connecting it to other subnetworks. The circuit is then re-assembled using these matrices to solve for the currents in the connecting components. One potential advantage is that we avoid handling the entire problem at once, and so reduce the size of arrays in a computer program from problem-sized to subnetwork-sized. Another advantage is that subnetwork results can be kept and used again without re-calculation in other problems containing the same 
subnetworks; this allows us to solve several similar problems more quickly than by complete re-solution for each problem.

\section{Diakoptic Solution}

The network is divided into subnetworks by boundary lines parallel to the coordinate axes between the lines of junctions (Fig. 1); the boundary lines need not run straight through the whole network. Every junction is within one subnetwork, but some of the circuit branches cross the boundary lines. We introduce as additional unknowns the currents $I_{R}$ in all these branches; these components are then taken out of the network, leaving a number of separate subnetworks. The removed branch currents $I_{R}$ appear as source currents for the subnetworks.

The first stage of computation is to analyse the internal behaviour of each section. For each junction within the subnetwork we have an equation of the form of Eq. (3). Some terms in this general form may be missing if the junction is next to a diakoptic boundary, or at the edge of the problem where the equation is modified to account for the boundary conditions. There may also be impressed source currents appearing on the right-hand side. All these equations are gathered into matrix form, with a suitable ordering of the junctions:

$$
\mathscr{C} \cdot \boldsymbol{V}=\boldsymbol{I}_{0}+\boldsymbol{I}_{R} .
$$

The matrix $\mathscr{C}$ contains all the equation coefficients (obtained from the circuit component values); it is symmetric and very sparse, having not more than five non-zero elements in each row or column. The vector $\boldsymbol{V}$ consists of all the unknown junction voltages. The right-hand side consists of current sources $I_{0}$ arising from boundary conditions at the edge of the problem, and also the currents $I_{R}$ in removed branches adjoining this subnetwork. In our computer program it is assumed that $\boldsymbol{I}_{0}$ is known at this stage, so that boundary conditions must be specified at the start. The currents $\boldsymbol{I}_{R}$ are not yet known, but the matrix $\mathscr{C}$ is completely determined.

Next we compute the inverse of $\mathscr{C}$. We have used a special method designed for sparse matrices, originated by ZOLLENKOPF (1971). This method stores only the non-zero elements of the matrix, with pointer arrays to locate them; it also obtains the inverse in a factored form which reduces the number of non-zero coefficients that must be stored.

The second stage is to construct a matrix $\mathscr{S}$ which describes the subnetwork from outside. Viewing the subnetwork as a black-box circuit with external terminals at the junctions where removed branches were attached, the matrix $\mathscr{S}$ relates the currents $I_{R}$ and the voltages $V_{R}$ at these terminals:

$$
\boldsymbol{V}_{R}=\boldsymbol{V}_{0}+\mathscr{S} \cdot \boldsymbol{I}_{R}
$$

$V_{0}$ is the vector of terminal voltages when none of the removed branches is carrying any current; they arise from sources representing boundary conditions at those edges of the problem included in this subnetwork. Each column of $\mathscr{S}$ consists of the terminal voltages when just one of the terminal currents is unity, and the rest are zero, and the problem boundary sources are removed. These terminal voltages are obtained using the inverse 
matrix $\mathscr{C}^{-1}$; from Eq. (4) we have:

$$
\boldsymbol{V}=\mathscr{C}^{-1} \cdot \boldsymbol{I}_{0}+\mathscr{C}^{-1} \cdot \boldsymbol{I}_{R}
$$

Comparing Eq. (5) and (6) we can identify $\boldsymbol{V}_{0}$ with $\mathscr{C}^{-1} \cdot \boldsymbol{I}_{0}$. Also each column of $\mathscr{S}$ is then $\mathscr{C}^{-1} \cdot \boldsymbol{I}_{R}$, where $\boldsymbol{I}_{R}$ has one unit element and the rest zero. We carry out a series of such matrix multiplications making each element of $\boldsymbol{I}_{R}$ unity in turn, to obtain all the columns of $\mathscr{S}$. In all these matrix multiplications we throw away the voltages at the internal junctions of the subnetwork, which result from the calculations at the same time as the terminal voltages. Because $\mathscr{C}^{-1}$ is only held in a factored form, actual matrix multiplications must be performed here, rather than just selecting terms out of an explicitly known matrix, which the multiplications are equivalent to.

Computations on the individual subnetwork are now complete. We save for future use the following information about the subnetwork: (a) the removed branch impedances $Z_{R}$, (b) the black-box matrix $\mathscr{S}$, (c) the terminal voltages $V_{0}$, (d) a record of problem boundary sources within the subnetwork, and (e) the inverse matrix $\mathscr{C}^{-1}$. Each other subnetwork is then analysed similarly.

The final stage is to re-assemble the network. We set up a network consisting of the black-box circuits of each subnetwork, joined together by the removed components. Apply Ohm's law to each removed component:

$$
I_{R}=\left(V_{R 1}-V_{R_{2}}\right) / Z_{R},
$$

where $V_{R 1}$ and $V_{R 2}$ are the terminal voltages at the ends of this component. Then we eliminate the voltages from this equation, using Eq. (5). The result is a set of linear equations relating the removed branches currents $I_{R}$ to each other, which we write schematically in the form:

$$
\boldsymbol{I}_{R}=\left\{\left(\boldsymbol{V}_{01}+\mathscr{S}_{1} \cdot \boldsymbol{I}_{R 1}\right)-\left(\boldsymbol{V}_{02}+\mathscr{S}_{2} \cdot \boldsymbol{I}_{R 2}\right)\right\} / Z_{R} .
$$

Each current is related to all other currents that border the two subnetworks it connects. In practical cases the matrix of coefficients of these equations is reasonably full, and we solve it by a standard elimination method for full matrices. Considerable effort is needed in setting up these equations, and elsewhere, to keep track of the connections of the various removed components and to be consistent in sign conventions for the currents.

Having solved these equations and obtained all the removed branch currents, we can select those that are relevant to a particular subnetwork, and substitute them in Eq. (6) to obtain all the junction voltages. When this is done for all subnetworks, the solution of the problem is complete.

\section{Numerical Example}

A computer program in FORTRAN has been written to use the diakoptic method. As an example we chose a simple geophysical model that one of us has used as an example in a previous paper (BREWITT-TAYLOR and WEAVER, 1976). Since diakoptics is a new method of solving the numerical equations only, and not a new method of setting them up, the solutions are exactly the same as those obtained in the previous paper, and so are not shown again here. The problem is shown in Fig. 2. It was solved for the $E$-polari- 


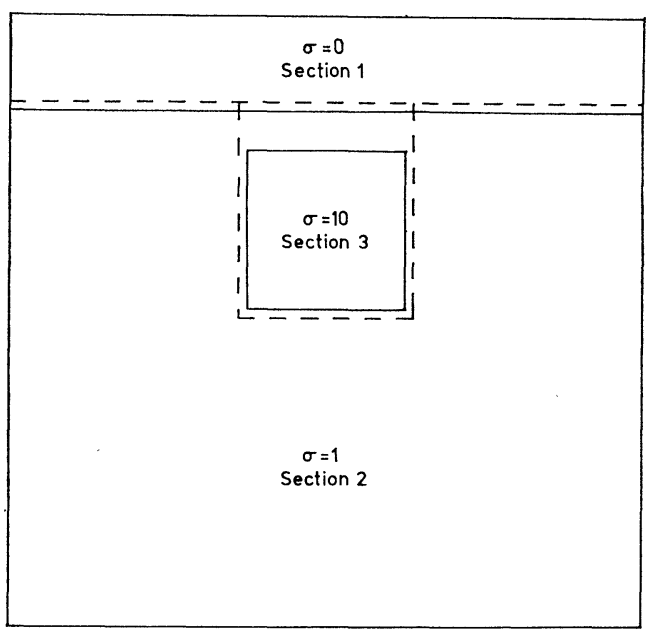

Fig. 2. The example problem. The solid lines show conductivity boundaries, the dashed lines show subnetwork boundaries.

sation case. The problem was first solved without division into subnetworks, using the same sparse-matrix inversion technique mentioned above; this is easily achieved in the program by omitting the steps relating to removed branches. It was then solved again by division into three subnetworks, as shown by the dashed lines in Fig. 2. The first subnetwork, representing the atmosphere, would only need solving once for any $E$ polarisation problem with this frequency and horizontal mesh spacing. The second subnetwork, representing a uniform region around the anomalous region, can be used for many anomalies. These are then obvious candidates for saving their subnetwork results for repeated use. The anomalous conductivity is confined within the third subnetwork, which will need to be re-computed for each anomaly used. In seeking to match an observed surface field distribution, it will be useful to try many such anomalous conductivities. With this subdivision one can use any anomaly that can be represented in the grid mesh in the third subnetwork, not necessarily the uniform block used here.

Computer times and storage estimates are shown in Table 1. The storage used depends greatly on how much programming effort is put into use of disc storage to save core storage. Subdivision gives a storage saving, as we avoid handling the very large inverse matrix for the whole problem. Looking at the computer times, we see that there is a considerable overhead in the extra computation of the diakoptic method, particularly

Table 1. Computer time and storage for the example problem.

\begin{tabular}{lcc}
\hline \multicolumn{1}{c}{ Operation } & Computer time & Storage \\
\hline Section 1 (air) & $14 \mathrm{sec}$ & $14 \mathrm{~K}$ \\
Section 2 (surrounds) & 134 & $59 \mathrm{~K}$ \\
Section 3 (anomalous) & 7 & $8 \mathrm{~K}$ \\
Joining sections & 13 & $27 \mathrm{~K}$ \\
Total for first problem & 168 & $59 \mathrm{~K}$ \\
Subsequent problems & 20 & $27 \mathrm{~K}$ \\
Undivided solution & 71 & $78 \mathrm{~K}$ \\
\hline
\end{tabular}

Storage is in units requiring one for an integer and two for a real number; the figures do not include storage for arrays that are independent of the subdivision (10 K in this case). 
in analysing the two large subnetworks. Much of this is spent doing the many matrix multiplications involved in obtaining the matrix $\mathscr{S}$. Thus for a single problem it is quicker to solve it without subdivision. To change the anomalous conductivity, however, we only need to analyse the small third subnetwork and re-assemble the problem, which in this example takes less than a third of the time of a complete re-solution. Thus we only need to solve a few anomalous conductivities before we have made a time saving as compared with complete undivided re-solution for each problem. The time taken for subnetwork assembly depends largely on the total number of removed branches; so for a quick solution of repeated problems it is desirable to minimise this number, and also the size of the subnetwork containing the anomaly. A compromise has to be reached between this consideration and the large time and storage required by large non-anomalous subnetworks and the flexibility provided by the subnetwork structure. Thus for this type of problem it would be quickest to use only two subnetworks (the anomalous region, and everywhere else), but this has the disadvantage that the normal subnetwork would need re-analysing for every change of surrounding medium, which would be a long process.

\section{Conclusion}

The diakoptic method provides a computer storage saving in any induction problem, at the expense of extra computation time. In the case of several problems in which only a small area of conductivity changes from one problem to the next, such as arise naturally in the interpretation of geophysical data, the method gives solutions to problems after the first considerably more quickly than repeated solution without subdivision. The solutions obtained are exactly the same as solutions of the same difference equations by more direct methods.

There is no need, of course, to express the problem in terms of an electrical network and the method of diakoptics or substructures may certainly be applied to the simultaneous equations assembled by the differencing process. However, in forming the substructures there is much more meaning in cutting a graph which corresponds directly to the physical problem rather than cutting a matrix which relates to the physical problem in a much less direct way. Thus there is considerable advantage in forming a graph. The use of electrical terms to describe the graph is merely a matter of convenience of language for the authors.

This work was carried out as part of a research contract from the U. K. Ministry of Defence.

\section{REFERENCES}

Brameller, A., M. N. John, and M. R. Scott, Practical Diakoptics for Electrical Networks, 242 pp., Chapman and Hall, London, 1969.

Brewitt-TAYLOR, C. R. and J. T. WeAver, On the finite difference solution of two-dimensional induction problems, Geophys. J. R. Astr. Soc., 47, 375-396, 1976.

Kron, G., Diakoptics, MacDonald, London, 1963.

SwIFT, C. M., Theoretical magnetotelluric and turam response from two-dimensional inhomogeneities, Geophysics, 36, 38-52, 1971.

Zollenkopf, K., Bi-factorization-Basic computational algorithm and programming techniques, in Large Sparse Sets of Linear Equations, edited by J. K. Reid, pp. 75-96, Academic Press, London, 1971. 